\title{
Philosophiques
}

\section{Le contextualisme sémantique en perspective} Au sujet de Literal Meaning, de François Recanati

\section{Michel Seymour}

Volume 33, numéro 1, printemps 2006

Philosophie et psychopathologie

URI : https://id.erudit.org/iderudit/012957ar

DOI : https://doi.org/10.7202/012957ar

Aller au sommaire du numéro

Éditeur(s)

Société de philosophie du Québec

ISSN

0316-2923 (imprimé)

1492-1391 (numérique)

Découvrir la revue

Citer ce document

Seymour, M. (2006). Le contextualisme sémantique en perspective : au sujet de Literal Meaning, de François Recanati. Philosophiques, 33(1), 249-262.

https://doi.org/10.7202/012957ar d'utilisation que vous pouvez consulter en ligne.

https://apropos.erudit.org/fr/usagers/politique-dutilisation/ 


\title{
Le contextualisme sémantique en perspective.
}

\section{Au sujet de Literal Meaning, de François Recanati}

\author{
MICHEL SEYMOUR \\ Université de Montréal \\ seymour@videotron.ca
}

François Recanati a produit un beau livre, finement ciselé sur le plan conceptuel, et très richement documenté. Il s'agit d'une argumentation généreuse pour le lecteur, et l'ouvrage se prête bien à une disputatio pour cette raison. Sa contribution s'inscrit dans une discussion récente qui, sous le couvert d'un débat entre spécialistes, réactive rien de moins que la vieille querelle opposant les sémanticiens formalistes et les tenants de la philosophie du langage ordinaire, et plus spécifiquement les théories anti-psychologistes et les théories psychologistes de la signification. J'explique dans un premier temps et très succinctement en quoi consiste le contextualisme de Recanati, et je procède ensuite à l'examen de l'argument justifiant cette théorie.

Tel que je le comprends, le contextualisme sémantique affirme la nécessaire dépendance naturelle de la signification littérale par rapport à la signification intentionnelle. Il ne faut pas comprendre cette théorie comme découlant d'un argument logique ou analytique. Il ne s'agit pas de prétendre que le concept de signification conventionnelle se réduit au concept de signification intentionnelle. Appelons cette dernière thèse "l'intentionnalisme ». On retrouve un argument logique et conceptuel de ce genre chez des auteurs comme Paul Grice, Stephen Schiffer, David Lewis, Jonathan Bennett et Gilbert Harman ${ }^{1}$. Le contextualisme cherche plutôt à établir une relation de nécessité naturelle entre deux phénomènes. Il ne s'agit pas d'une nécessité conceptuelle ou d'une nécessité purement métaphysique, mais bien d'une nécessité qui s'impose étant donné les faits contingents qui caractérisent notre condition.

Il ne faut pas non plus confondre la thèse contextualiste et les faits empiriques à partir desquels cette thèse est affirmée. On peut être appelé à reconnaître les faits empiriques qui viennent appuyer la thèse, et donc à reconnaître l'existence d'une corrélation effective entre les conditions de vérité intuitives des énonciations et la psychologie des locuteurs, sans tirer la conclusion qu'il existe une relation de nécessité naturelle entre la significa-

1. Paul Grice, " Meaning », Philosophical Review, 66, 1957 ; Grice, « Utterer’s Meaning, Sentence Meaning and Word-Meaning ", Foundations of Language, 4, 1968 ; Grice, "Utterer's Meaning and Intentions », Philosophical Review, 78, 1969 ; Grice, " Meaning Revisited » dans N. Smith (dir.), Mutual knowledge, London, Academic Press, 1982 ; David Lewis, Convention : A Philosophical Study, Harvard University Press, 1969 ; Stephen Schiffer, Meaning, Oxford, Clarendon Press, 1972 ; Jonathan Bennett, Linguistic Behavior, Cambridge, Cambridge University Press, 1976 ; Gilbert Harman, " Review of Linguistic Behavior by Jonathan Bennett ", Language, 1977, 53. 
tion littérale et la signification intentionnelle. Les données empiriques viennent sans doute corroborer l'hypothèse contextualiste, ou à tout le moins lui fournissent-elles un certain degré de plausibilité, mais la postulation d'un lien de nécessité naturelle constitue un aspect plus controversé du contextualisme. Il s'agit d'une certaine façon d'expliquer les phénomènes observés.

Par exemple, un auteur comme Ludwig Wittgenstein serait tout à fait disposé à reconnaître l'importance des facteurs pragmatiques pour la détermination des conditions de satisfaction des énonciations, et il serait en ce sens disposé aussi à reconnaître les prémisses empiriques du contextualisme. Mais il le ferait tout en défendant fermement l'autonomie de l'institution du langage, autant sur le plan conceptuel que du point de vue de la nécessité naturelle. Autrement dit, il récuserait autant la thèse intentionnaliste que la thèse de la nécessité naturelle défendue par les philosophes contextualistes, tout en donnant largement raison à ces derniers au sujet des hypothèses empiriques qu'ils avancent puisque, très souvent, les conditions intuitives de satisfaction des énonciations s'éloignent considérablement des conditions de vérité déterminées par la signification conventionnelle des mots.

La même remarque s'applique à Herman Cappelen et Ernest Lepore ${ }^{2}$. Ces deux auteurs sont disposés à reconnaître le pluralisme des actes de langage, c'est-à-dire l'idée qu'une quantité très grande de propositions peuvent être dites, assertées, affirmées à l'occasion d'énonciations particulières d'une seule et même phrase. Ils sont donc d'accord pour dire que, dans le contexte d'une énonciation donnée, les conditions de vérité intuitives sont dérivées à partir des contenus d'intention de signifier et des présuppositions. Ce qui est dit dans un contexte dépend de facteurs très variés qui diffèrent de la proposition sémantiquement exprimée. En somme, ils acceptent que les actes de langage expriment potentiellement une quantité très grande de propositions. Mais qu'à cela ne tienne, le pluralisme des actes de langage est compatible avec le minimalisme sémantique, c'est-à-dire avec le point de vue affirmant (i) que peu d'expressions signifient en fonction du contexte, (ii) que celles qui le font sont grammaticalement sensibles au contexte et (iii) que le contexte d'énonciation n'a pas d'incidence sur le contenu littéral exprimé par les expressions du langage. Or le philosophe contextualiste refuse d'expliquer la dépendance observée entre les conditions de satisfaction des énonciations et les intentions de signifier sans que cela n'ait d'impact sur le plan de la signification littérale. Pour lui, cette dépendance observée est révélatrice d'une dépendance plus profonde entre le contenu littéral et le contenu intentionnel.

J'ai distingué les thèses intentionnaliste et contextualiste. Celles-ci peuvent, bien entendu, être développées de manière convergente, la raison étant que les deux permettent de développer ensemble une théorie psychologiste de la signification. C'est notamment ce qui se produit chez John Searle. L'hypothèse

2. Voir «A Tall Tale : In Defense of Semantic Minimalism and Speech Act Pluralism» dans Gerhard Preyer (dir.) Contextualism, Oxford, Oxford University Press, 2004 ; voir surtout leur ouvrage Insensitive Semantics, Basil Blackwell, 2004. 
de l'arrière-plan joue chez Searle un rôle important dans la détermination des conditions de vérité littéralement attachées aux énoncés, ce qui fait de lui un philosophe contextualiste ${ }^{3}$. Mais la dérivation proposée de la notion d'institution du langage à partir d'un ensemble de concepts psychologiques (l'arrière-plan, le système de croyances, les intentions collectives) place également Searle du côté des intentionnalistes ${ }^{4}$.

Il importe toutefois de souligner le fait que l'intentionnalisme et le contextualisme ne sont pas nécessairement des thèses convergentes. Certains intentionnalistes rejettent en principe le contextualisme. Paul Grice, par exemple, pense que dans un contexte d'énonciation donné, la part la plus importante de la signification est constituée par la signification conventionnelle attachée aux mots, et il soutient que les facteurs pragmatiques ne viennent que très rarement troubler le contenu sémantique véhiculés. Ainsi, il a tendance à minimiser l'importance des facteurs pragmatiques dans la détermination des conditions de vérité littérales attachées à une énonciation donnée. On peut défendre une telle position minimaliste eu égard aux ingrédients pragmatiques requis dans le contexte d'une énonciation particulière, tout en adoptant sur le plan conceptuel une approche réductionniste au sujet des conventions gouvernant les règles qui donnent la signification invariante des mots.

Inversement, on peut être contextualiste sans être intentionnaliste. Il existe, en effet, plusieurs variantes de l'hypothèse contextualiste. Certains auteurs en viennent à nier l'existence même d'une composante conventionnelle de la signification des mots. On songe ici à ceux pour qui la signification d'un mot n'existe que dans l'usage entendu au sens d'événement d'énonciation. On peut qualifier cette position de contextualisme radical. Si l'on s'engage dans cette voie, le contextualisme n'a pas besoin d'une thèse intentionnaliste pour aboutir au psychologisme. Il y parvient tout seul. Il est donc possible, dans une telle perspective, d'aller jusqu'à rejeter l'hypothèse intentionnaliste. Mais la plupart des auteurs reconnaissent l'existence d'un sens conventionnel (ou d'un potentiel sémantique) attaché aux mots, tout en soutenant que les conditions de vérité déterminées par ceux-ci ne jouent souvent aucun rôle dans la détermination des conditions de vérité littérales associées aux énoncés. Il s'agit dans ce cas du contextualisme modéré. Par conséquent, même si le contextualisme peut en principe aboutir par lui-même au psycholo-

3. John Searle, «Literal Meaning », Erkenntnis 1, 1978, 207-224 (reproduit dans Searle, Expression and Meaning : Studies in the Theory of Speech Acts, New York, Cambridge University Press, 1979) ; Searle, Intentionality : An Essay in the Philosophy of Mind, New York, Cambridge University Press, 1983 ; Searle, "Response : The Background of Intentionality and Action ", dans Lepore et Van Gulick, 1991 ; Searle, The Rediscovery of the Mind, Cambridge, Mass., MIT Press, 1992.

4. Searle, The Construction of Social Reality, New York, Free Press, 1995.

5. Le minimalisme est défendu par Grice notamment dans «The Causal Theory of Perception ", PAS, suppl. vol. 35, 1961 ; "Logic and Conversation ", dans Peter Cole et Jerry L. Morgan (dir), Syntax and Semantics, vol. 3, Speech Acts, New York, Academic Press, 1975 ; Studies in the Way of Words, Cambridge, Mass., Harvard University Press, 1989. 
gisme, la version la plus plausible requiert sans doute des appuis additionnels, dont celui de l'intentionnalisme.

Dans le cadre de cette présentation, je ne discute pas de la théorie psychologiste de la signification en général, et je ne discute pas de l'intentionnalisme. J'examine seulement le contextualisme, et je concentre mon attention sur la version modérée défendue par des auteurs tels que Robyn Carston, John Searle, Dan Sperber et Deirdre Wilson ${ }^{6}$, et non sur la version radicale que l'on trouve chez des auteurs tels que Charles Travis, Peter Hacker et Gordon Baker ${ }^{7}$. La version modérée est aussi la version défendue par François Recanati dans Literal Meaning. Je veux spéculer sur les zones d'ombre qui me sont apparues à la lecture de ce livre ${ }^{8}$.

Le contextualisme de François Recanati affirme l'existence d'une relation de nécessité naturelle entre la signification littérale et des facteurs pragmatiques qui sont tout à la fois (i) primaires, (ii) optionnels et (iii) intentionnels. Les facteurs pragmatiques concernés sont primaires au sens où ils interviennent dans la détermination des conditions de vérité littérales de l'énoncé. On les distingue des facteurs pragmatiques secondaires qui viennent ajouter une couche additionnelle de signification (pragmatique) par rapport à la signification littérale. Songeons, par exemple, à l'ironie, à la métaphore, aux implicatures conversationnelles et aux actes de langage indirects qui présupposent des énoncés ayant déjà une signification littérale. On s'intéresse plutôt à des phénomènes qui interviennent dans la spécification des conditions de vérité littérales. Les facteurs pragmatiques pertinents sont aussi optionnels au sens où ils ne découlent pas des règles sémantiques associées aux expressions. Les expressions indexicales et les démonstratifs, par exemple, ont des règles de fonctionnement qui imposent sémantiquement le renvoi au contexte d'énon-

6. Robyn Carston « Enrichment and Loosening : Complementary Processes in Deriving the Proposition Expressed ?", Linguistiche Berichte 8, 1997, 103-127. Searle, "Literal Meaning ", op. cit. ; Dan Sperber et Deirdre Wilson, Relevance : Communication and Cognition, Oxford, Basil Blackwell, 1986.

7. Charles Travis, Saying and Understanding, Oxford, Basil Blackwell, 1975 ; Gordon P. Baker et P.M.S. Hacker, Wittgenstein, Rules, Grammar, and Necessity, Oxford, Basil Blackwell, 1985 ; Gordon P. Baker et P.M.S. Hacker, Scepticism, Rules and Language, Oxford, Basil and Blackwell, 1984.

8. Il existe aussi au moins deux variantes du contextualisme modéré. Certains (Recanati) peuvent reconnaître le fait que les expressions ont des significations conventionnelles pleines et entières qui contribuent partiellement à la signification des énoncés, même si elles ne peuvent pas toujours produire par elles-mêmes les conditions de vérité intuitives des énoncés. D'autres (Sperber et Wilson) diront que les expressions n'ont qu'un potentiel sémantique et que la signification qui leur est associée dans le cadre d'une énonciation donnée dépend en partie du potentiel sémantique, mais aussi en partie du contexte. La notion de potentiel sémantique peut servir à montrer que la part conventionnelle doit être enrichie par le contexte pour compléter la contribution sémantique de l'expression à l'énoncé. Cette notion rapproche grandement le philosophe contextualiste du contextualisme radical. Je ne discuterai pas de cette alternative en tant que telle, sauf à la toute fin de ce texte. 
ciation. Le contexte permet de compléter l'énoncé qui exprime alors des conditions de vérité pleines et entières. Il s'agit d'un phénomène de saturation à ne pas confondre avec le phénomène de modulation dont il est question ici, dans lequel des facteurs pragmatiques interviennent de manière facultative, mais sont néanmoins pertinents pour déduire les conditions de vérité littérales d'un énoncé donné. Les facteurs pragmatiques concernés sont enfin aussi décrits comme intentionnels au sens où il ne suffit pas de faire appel à une notion étroite de contexte n'impliquant rien de plus que le temps, le lieu, le locuteur, ainsi que des données proximales ou distales. Il faut faire intervenir les intentions de signifier et les croyances des locuteurs.

L'idée essentielle du contextualisme de Recanati est que les facteurs pragmatiques primaires, optionnels et intentionnels viennent parfois s'interposer entre les significations conventionnelles des mots et la signification des phrases. Les conditions de vérité littérales de plusieurs énoncés sont déterminées en partie par la signification conventionnelle des expressions constitutives, mais aussi par de tels facteurs pragmatiques. Les conditions de vérité qui découlent de la signification conventionnelle des mots ne sont pas toujours pertinentes. Sont parfois aussi pertinentes les conditions de vérité signifiées par le locuteur et auxquelles les autres locuteurs ont accès. Recanati soutient que divers phénomènes pragmatiques permettent d'illustrer ce point de vue. On songe à l'enrichissement (enrichment) [ex. : "il prit sa clé et ouvrit la porte "; on comprend qu'il l'a ouverte avec sa clé], au relâchement (loosening) [ex. : « le guichet automatique a avalé ma carte de débit »; ce que l'on comprend n'implique aucun phénomène de déglutition] et au transfert métonymique (transfer) [ex. : "le sandwich au jambon est parti sans payer "; on comprend que c'est le consommateur du sandwich qui est visé].

J'en viens finalement à mes commentaires. Je vais me concentrer sur un seul problème, mais il est d'importance, puisqu'il concerne l'argument en faveur du contextualisme. Je me suis demandé quel argument particulier Recanati entendait développer en faveur du contextualisme. En voici une première reconstruction. Les conditions de vérité d'un énoncé constituent sans doute l'un des ingrédients sémantiques par excellence. Or il arrive très souvent que les conditions de vérité d'une énonciation donnée requièrent pour leur spécification le recours à des facteurs pragmatiques, comme le sens visé par les locuteurs. Il y a donc un lien étroit entre la signification littérale et de tels facteurs pragmatiques apparaissant dans le contexte, d'où la conclusion que l'acte de langage est le véhicule premier de la signification. Cette version de l'argument est bien entendu non valide. Le fait que les conditions de vérité d'une énonciation donnée requièrent que l'on fasse intervenir des facteurs pragmatiques ne prouve aucunement qu'il existe un lien étroit entre la signification littérale et le contexte d'énonciation. Il y a sans doute très souvent un lien entre la signification d'une énonciation et des facteurs pragmatiques intentionnels, mais cela ne veut pas dire que la signification littérale de la phrase soit 
soumise aux mêmes contraintes. Le saut injustifié survient lorsque l'on tente de tirer une conclusion concernant les conditions de vérité littérales des phrases à partir de faits observés concernant les conditions de vérité de leurs énonciations. Herman Cappellen et Ernest Lepore sont les premiers à reconnaître qu'une seule et même phrase peut être énoncée pour accomplir différents actes de langage et exprimer des conditions de vérité variables d'un contexte à l'autre. Il ne s'en suit pas que les conditions de vérité littérales des phrases soient elles-mêmes déterminées par le contexte. Autrement dit, l'erreur est de partir des énonciations et de constater que leurs conditions de vérité sont liées à des facteurs pragmatiques, pour ensuite soutenir que les conditions de vérité des phrases elles-mêmes sont liées au contexte.

Une variante de cet argument semble être défendue par Recanati. Il s'agit cependant d'une variante faisant intervenir une prémisse additionnelle qui manquait à la première version et qui, pour cette raison, rend l'argument plus convaincant. En vertu du principe de disponibilité (availability principle), les conditions de vérité d'une phrase donnée doivent être celles qui sont accessibles aux individus (p. 20). Or les conditions de vérité auxquelles plusieurs individus accèdent à l'occasion de certaines énonciations données sont déterminées par des facteurs pragmatiques primaires, optionnels et intentionnels. La conclusion contextualiste semble alors découler de ces prémisses. Le principe de disponibilité nous offre ainsi la prémisse manquante de l'argument initialement formulé. C'est une prémisse qui justifie le saut nous incitant à assimiler les conditions de vérité littérales des phrases aux conditions de vérité intuitives des énonciations. On y parvient en imposant un principe de disponibilité. Cet argument laisse entendre que les conditions de vérité littérales doivent être disponibles, c'est-à-dire qu'elles doivent être celles qui sont effectivement appréhendées par les agents. Appelons ces dernières les conditions de vérité intuitives. Si les conditions de vérité littérales doivent être les conditions de vérité intuitives et que celles-ci sont fonction de facteurs pragmatiques primaires, optionnels et intentionnels, alors la signification dépend fondamentalement des événements d'énonciation, ou si l'on veut, des actes de langage. Mais pour remonter au point de départ de l'argument et bien identifier la source du différend, il faut poursuivre la réflexion et se demander quelle est la justification du principe de disponibilité et quel est son rôle exact dans l'argument.

Je suppose que l'on peut invoquer un principe méthodologique semblable à celui qui est proposé par Michael Dummett ${ }^{9}$ pour justifier le principe de disponibilité. Toute théorie de la signification doit en même temps être une théorie de la compréhension. Sur la base de ce principe général, on peut défendre des thèses corollaires plus particulières. On dira, par exemple, que toute théorie de la référence doit être complétée par une théorie du sens ${ }^{10}$. Et on ira peut-être ensuite jusqu'à affirmer que toute théorie du sens doit en même

9. Michael Dummett, "What is a Theory of Meaning ? I ", dans The Seas of Language, Oxford, Oxford University Press, 1996. 
temps se présenter comme une théorie du contenu. On se rapprocherait alors beaucoup du principe de disponibilité défendu par Recanati. Ce dernier principe suppose que les conditions de vérité littérales des phrases (le sens) doivent justement être celles qui sont comprises par les locuteurs (le contenu). Elles doivent être présentes dans le contenu cognitif des états mentaux des interlocuteurs à l'occasion d'une énonciation donnée et donc être des conditions de vérité intuitivement partagées par les locuteurs. Or, justement, les conditions de vérité intuitives auxquelles les locuteurs accèdent sont souvent fonction des intentions de signifier, alors que les prétendues conditions de vérité " littérales ", celles qui découlent seulement de la signification conventionnelle de leurs expressions constitutives, ne sont parfois même pas appréhendées par eux et ne jouent aucun rôle dans la compréhension de l'énonciation. D'où l'idée que la signification littérale est fonction du contexte d'énonciation.

Je dois dire que je ne suis pas du tout convaincu par les arguments de Recanati concernant la disponibilité des conditions de vérité des phrases. Comment peut-on soutenir que celui qui entend «Elle a sorti sa clé et ouvert la porte " et comprend qu'elle a ouvert sa porte avec la clé n'accède pas aux conditions de vérité de la phrase ? Ne faut-il pas dans ce cas les saisir pour pouvoir les enrichir ? De même, pour comprendre ce qui est dit dans "Le guichet automatique a avalé ma carte de débit ", ne faut-il pas imaginer un processus de déglutition pour opérer le relâchement ? Enfin, pour comprendre que le client lui-même est visé dans « Le sandwich au jambon est sorti sans payer ", ne faut-il pas partir de la situation insensée de l'état de choses représenté par les conditions de vérité de la phrase ? Pour Recanati, le malaise ressenti à l'écoute de cette phrase provient de l'incompatibilité entre le concept exprimé par le sujet et le concept exprimé par le prédicat. On ne se rend donc jamais au point où serait représenté un état de choses possible, par exemple un sandwich qui sort du restaurant. J'avoue que je ne partage pas ici les intuitions de Recanati, car le malaise ressenti à l'occasion d'une première écoute de la phrase semble s'expliquer justement par le fait que l'on ne voit pas pourquoi il est affirmé qu'un sandwich au jambon est littéralement sorti du restaurant. Après quelques secondes, cette suggestion disparait, et l'on comprend désormais qu'il est question du client. À partir de ce moment, les conditions de vérité littérales n'interviennent plus dans notre contenu cognitif. Mais il a fallu qu'elles interviennent initialement pour que puisse s'opérer un transfert métonymique.

Dans la suite de ce texte, je vais toutefois ignorer ce désaccord. Je vais faire comme si Recanati avait raison de dire que nous n'accédons pas toujours aux conditions de vérité découlant des significations conventionnelles des mots. Supposons donc qu'il ait raison sur ce point. Les conditions de vérité disponibles sont dans certains contextes d'énonciation des conditions de vérité intuitives qui s'éloignent de celles découlant de la signification conventionnelle des mots. 
Dans cette version de l'argument, on est quand même passé subtilement d'une prescription méthodologique à une hypothèse plus controversée affirmant que le sens doit être précisément ce qui intervient dans le contenu cognitif des agents. C'est ainsi que l'on est en position de justifier ensuite une assimilation des conditions de vérité littérales des phrases aux conditions de vérité intuitives saisies par les locuteurs à l'occasion d'une énonciation, en conformité avec le principe de disponibilité. On aurait pourtant pu penser que la prescription méthodologique nous enjoignait seulement d'inclure une théorie de la compréhension en plus d'une théorie de la signification. Le principe méthodologique de Dummett peut pour cette raison être compatible avec une thèse bifurcationniste ${ }^{11}$ qui aurait à spécifier des conditions de vérité littérales exprimées par les phrases et les conditions de vérité intuitives des énonciations, tout en montrant les liens qui existent entre ces deux sortes d'ingrédients au sein d'une théorie unitaire. Si l'on souscrit au principe méthodologique de Dummett, la théorie de la signification doit être en même temps une théorie de la compréhension, mais cela peut se faire sans avoir à produire un ingrédient qui est simultanément un sens et un contenu. Pour procéder de la sorte en respectant le principe de disponibilité, il suffirait que les conditions de vérité découlant de la signification conventionnelle des mots soient en principe elles aussi accessibles, même si elles ne sont pas toujours celles auxquelles on accède dans tous les contextes d'énonciation.

Pour identifier encore plus précisément la source du différend, notons que le principe de disponibilité peut recevoir une interprétation faible et une interprétation robuste. Selon l'interprétation faible, il faut seulement que le contenu sémantique soit en principe accessible pour être un contenu exprimé. Même si, dans certains contextes d'énonciation, il n'intervient pas dans les contenus mentaux des agents, on le fait intervenir en tant que tel dans d'autres contextes. Alors, en vertu de la version faible du principe de disponibilité, on devrait admettre que les conditions de vérité des phrases sont disponibles même dans les contextes où les locuteurs les contournent sans les appréhender. Selon l'interprétation robuste, on n'imposerait pas seulement une condition nécessaire, mais aussi d'une certaine façon une condition suffisante. Il ne s'agirait pas simplement d'affirmer que le contenu sémantique d'un énoncé doit en même temps être en principe accessible. On prétendrait que ce à quoi l'on accède dans un contexte d'énonciation donné constitue d'emblée le contenu sémantique. Or il semble que ce soit en s'appuyant sur un principe de disponibilité compris au sens robuste que l'on est en mesure de dire que les conditions de vérité littérales ne sont rien de plus que celles qui sont saisies intuitivement par les locuteurs. On ne peut plus se contenter de parler de conditions de vérité littérales exprimées par les phrases qui seraient en principe accessibles (le sens), et auxquelles viendraient ensuite s'ajouter des conditions de vérité de l'énonciation spécifiant ce qui est compris par les locuteurs dans le contexte (le contenu). Le principe de disponibilité, interprété dans sa version robuste, permet d'interdire tout bifurcationnisme, car on semble 
alors justifié d'imposer une seule entité qui agirait à la fois comme sens et contenu dans tous les contextes d'énonciation. Donc, selon lui, si j'énonce que Recanati a sorti ses clés et ouvert la porte, les conditions de vérité littérales doivent être celles qui supposent qu'il s'est servi de ses clés pour ouvrir la porte, et ce, même si la phrase proprement dite semble exprimer des conditions de vérité plus pauvres.

Recanati ne fait pas au départ intervenir son principe de disponibilité comme une condition suffisante ; il s'agit dans un premier temps d'une condition nécessaire, d'une « contrainte » que les conditions de vérité doivent satisfaire. Et il n'affirme pas que ce qui est saisi intuitivement dans un contexte constitue en même temps le contenu littéral. Recanati affirme seulement que ce qui est dit doit être intuitivement accessible aux participants à la conversation (p. 20). Toutefois, en même temps, la contrainte doit s'appliquer à ce qui se produit à l'occasion d'une énonciation. Il est affirmé que « ce qui est dit doit être analysé en conformité avec les intuitions partagées par ceux qui comprennent parfaitement l'énonciation" (p. 14, c'est moi qui souligne). Si, dans un contexte d'énonciation donné, les conditions de vérité intuitives ne coïncident pas avec les conditions de vérité minimales, à savoir celles qui découlent de la signification conventionnelle des mots, mais sont les seules à être appréhendées par les locuteurs, alors, selon Recanati, les conditions de vérité littérales ne sont rien de plus que les conditions de vérité intuitives. Donc, même si le principe de disponibilité est présenté au départ seulement comme une condition nécessaire, le fait qu'on se concentre exclusivement sur ce qui se produit à l'occasion d'énonciations particulières est ce qui permet à Recanati de dire que le contenu littéral est parfois identique au contenu intuitif saisi par les locuteurs. Or l'accessibilité est pour lui une notion intimement liée aux événements d'énonciation (pp. 30-32). Il s'en tient à ce qui se produit quant aux énonciations, et c'est de cette manière qu'il arrive à rabaisser parfois le contenu littéral au contenu intuitif. Les conditions de vérité minimales ne sont-elles pas elles aussi parfois accessibles ? Bien sûr qu'elles le sont, et elles sont alors pour cette raison en principe accessibles dans tous les contextes d'énonciation. Cependant, le fait d'appliquer le principe de disponibilité aux énonciations nous permet apparemment de les exclure parfois du contenu littéral.

Une hypothèse de rechange, opposée au principe de disponibilité, semble susceptible d'être proposée. On prétendrait que même si les locuteurs ne font pas toujours intervenir de manière occurrente les conditions de vérité découlant de la signification conventionnelle des mots, ils ont aussi accès de manière générale à de telles conditions de vérité minimales. Cela serait compatible avec le fait que, dans plusieurs contextes d'énonciation, les conditions de vérité minimales ne coïncident pas avec les conditions de vérité intuitives. Ces autres cas ne fourniraient pas de raison pour évacuer les conditions de vérité minimales de la signification littérale. Les énoncés continuent d'exprimer ces conditions 
de vérité minimales, y compris dans les contextes où les locuteurs ne les font pas intervenir dans leur esprit.

Le philosophe contextualiste ne voit pas les choses de la même façon. Il y a des contextes d'énonciation dans lesquels les conditions de vérité littérales coïncident avec les conditions de vérité minimales (celles qui découlent de la signification conventionnelle des expressions), et des contextes d'énonciation dans lesquels les conditions de vérité littérales coïncident avec les conditions de vérité plus riches, celles qui font intervenir des facteurs pragmatiques additionnels. Mais justement, puisqu'il nous faut reconnaître ce dernier cas, il faut aussi reconnaître la thèse essentielle de la dépendance au contexte.

Si le philosophe contextualiste est prêt à s'engager dans une telle voie argumentative, je crains que, là encore, il y ait un problème important. Comme on vient de le voir, il semble que les conditions de vérité « littérales » soient toujours, selon le philosophe contextualiste, les conditions de vérité exprimées par les énoncés dans un contexte d'énonciation. Nous avons vu que dans un contexte où des facteurs pragmatiques primaires, optionnels et intentionnels ne surviennent pas, les conditions de vérité littérales ne sont, selon Recanati, rien de plus que les conditions de vérité minimales, alors que dans d'autres contextes, il s'agit de conditions de vérité plus riches. Or c'est seulement parce que l'on fait du contexte d'énonciation le médium ultime de la signification que les conditions de vérité littérales ne coïncident pas toujours avec les conditions de vérité minimales. Le principe de disponibilité serait en principe compatible avec une hypothèse bifurcationniste si l'on ne sous-entendait pas que tout dépend des énonciations. Or, si le contexte d'énonciation est de cette manière jugé déterminant pour établir ce qui doit compter comme conditions de vérité littérales, l'argument contextualiste semble circulaire. La conclusion à laquelle on voulait parvenir n'était-elle pas justement que les actes de langage étaient les véhicules premiers de la signification ? Et n'est-ce pas cette conclusion que l'on est en train d'affirmer implicitement dans les prémisses de l'argument lorsqu'on soutient que les conditions de vérité littérales sont déterminées en fonction du contexte d'énonciation ? N'est-on pas en train d'affirmer que le contexte d'énonciation est déterminant et qu'il est le lieu où se déploie la signification linguistique ? Pourquoi devrions-nous en rester aux énonciations pour établir ce qui est exprimé littéralement dans les phrases ? N'est-ce pas justement parce que, pour Recanati, les actes de langage sont les véhicules premiers de la signification ? Et le problème n'est-il pas alors que l'on présuppose ce qui devait être la conclusion de l'argument ? Encore une fois, les conditions de vérité minimales sont en principe accessibles. Mais dans des énonciations données, elles ne sont apparemment pas ce à quoi les locuteurs accèdent. Si l'on se croit en droit de les évacuer parfois de la signification littérale, c'est que l'on prend les événements d'énonciation comme étalon de mesure. Mais cela revient à faire entrer la conclusion désirée dans les prémisses de l'argument. 
Selon le point de vue bifurcationnsiste, les phrases ont un contenu sémantique minimal, et les énonciations ont des conditions de vérité qui sont très souvent plus riches et différentes du contenu sémantique minimal. Les conditions de vérité littérales exprimées dans tous les contextes sont celles qui découlent de la signification conventionnelle des expressions et sont elles-mêmes minimales. Elles satisfont le principe de disponibilité parce qu'elles sont toutes en principe accessibles, même si dans certains contextes d'énonciation, les locuteurs n'y ont apparemment pas accès. Toutefois, à cause, justement, du caractère minimal du contenu littéral exprimé, l'on se sert très souvent dans un contexte d'énonciation donné de ces mêmes énoncés pour dire des choses supplémentaires ou différentes. Toute énonciation permet alors de véhiculer un contenu plus riche, mais il ne faut pas s'appuyer sur ce fait pour tirer des conclusions erronées sur les liens qui existent entre la signification littérale des phrases et la signification contextuelle. On peut admettre que les phrases véhiculent un contenu sémantique minimal, auquel vient s'ajouter une couche pragmatique dans presque tous les contextes d'énonciation. Les conditions de vérité minimales qui découlent de la signification linguistique autonome des expressions sont peut-être parfois des conditions de vérité pauvres, mais elles sont des conditions de vérité quand même. On n'y a peut-être pas toujours recours, au sens où l'on ne les fait pas toujours intervenir dans notre esprit dans tous les contextes d'énonciation, mais ces conditions de vérité n'en existent pas moins. Il ne faut pas abandonner cette composante sous prétexte qu'elle ne nous serait souvent d'aucun recours dans une énonciation donnée.

Je viens de suggérer que la conception bifurcationniste est compatible avec le principe faible de disponibilité et qu'elle est préférable à l'hypothèse envisagée par Recanati, car celui-ci doit, pour défendre la version robuste du principe, s'appuyer sur un argument circulaire. Toutefois, un autre argument peut être envisagé en faveur du philosophe contextualiste, et qui explique peutêtre pourquoi il est impossible d'envisager des conditions de vérité découlant de la signification conventionnelle des mots, lesquels auraient une relative autonomie par rapport au sens visé par les locuteurs. Selon ce point de vue, les conditions de vérité minimales sont indéterminées et appellent à être complétées par l'inclusion de facteurs pragmatiques primaires, optionnels et intentionnels. Il en est ainsi parce que la signification des énoncés doit être déterminée. Puisqu'elle ne peut pas être toujours déterminée par les conditions de vérité de la phrase elle-même, elle doit l'être par le sens visé par les locuteurs dans le contexte. Ainsi, on arrive à la même conclusion d'une nécessaire dépendance de la signification littérale par rapport aux intentions de signification, mais on y parvient sans recourir cette fois-ci à un argument circulaire. L'argument requiert toutefois que la signification soit conçue comme déterminée. Certains passages du texte laissent entendre que Recanati est tenté par cette approche. Il écrit : "Par «contextualisme», j'entends le point de vue selon lequel ce sont les actes de langage, et non les phrases, qui ont un con- 
tenu déterminé et sont susceptibles d'avoir une valeur de vérité : les phrases elles-mêmes expriment un contenu déterminé seulement dans le contexte d'un acte de langage » (p. 154).

Ici, on éviterait la circularité, mais on n'éviterait pas de se compromettre sur une question pour le moins controversée, à savoir la thèse portant sur le caractère déterminé de la signification. Indépendamment de cette difficulté, il y a aussi le fait que l'on a reconnu le caractère optionnel des facteurs pragmatiques. Puisque ces facteurs n'interviennent pas toujours, ne doit-on pas admettre que les conditions de vérité minimales sont parfois les seules à être pertinentes, et ce, même si le contenu exprimé est alors indéterminé ? Je soutiendrais alors qu'il existe une tension entre, d'une part, une éventuelle adhésion au caractère déterminé de la signification et, d'autre part, l'admission que les mots ont une signification autonome et que les facteurs pragmatiques sont optionnels. Ces deux dernières thèses semblent conduire à une approche qui admet l'indétermination dans certains cas. Je doute par conséquent que le philosophe contextualiste puisse se permettre d'avancer que la signification doit être déterminée dans tous les cas. Il peut difficilement se dispenser des conditions de vérité littérales, que j'ai appelées "conditions de vérité minimales ", s'il est prêt à reconnaître à la fois que les mots ont des significations conventionnelles autonomes, et que les facteurs pragmatiques déterminants dont il est question sont réellement optionnels.

Certains philosophes croient que l'admission de l'indétermination radicale affectant toutes les expressions du langage, y compris les mots, nous conduit irrémédiablement dans les bras du contextualisme. Cet argument présuppose très souvent que le contenu des énoncés doit être déterminé. Si les expressions sont indéterminées et que le contenu doit être déterminé, alors il ne peut l'être que par des facteurs pragmatiques provenant de l'énonciation. La thèse de l'indétermination de la signification des mots et des phrases viendrait en ce sens appuyer le contextualisme, mais ce serait seulement parce qu'on sous-entendrait que le contenu doit être déterminé. Son caractère déterminé ne pourrait alors être expliqué que par l'adjonction de facteurs pragmatiques additionnels. Mais je viens de neutraliser cet argument en montrant que le philosophe contextualiste est lui-même engagé à reconnaître que, parfois, les conditions de vérité minimales et indéterminées sont les seules qui soient pertinentes. On ne peut pas non plus tenter d'exploiter le fait que, dans certains contextes d'énonciation, certains énoncés appellent à être déterminés. Car si tel est le cas, il s'agit tout au plus d'une exigence pragmatique qui s'impose en fonction du sens visé par le locuteur, et non pas en fonction de la signification littérale. Pire, cela revient encore une fois à faire du contexte d'énonciation l'étalon de mesure de ce qui est signifié littéralement, ce qui nous replace dans le cadre d'une argumentation circulaire.

Si les conditions de vérité littérales sont parfois les conditions de vérité minimales, on ne peut alors plus invoquer un principe général nous enjoignant de compléter les conditions de vérité minimales par des conditions de vérité 
plus riches. On peut seulement affirmer que les locuteurs saisissent parfois un contenu prétendument " déterminé " dans certains contextes d'énonciation. Toutefois, comme on l'a dit et répété, la dépendance de la signification d'une énonciation par rapport au sens visé par les locuteurs ne nous permet pas de prouver quoi que ce soit quant à la signification des phrases. Il est par conséquent permis de penser que les conditions de vérité minimales ont d'une manière générale une relative autonomie, et ce, y compris dans les contextes d'énonciation dans lesquels les locuteurs visent des significations plus riches. Ne faut-il pas alors reconnaître deux niveaux de signification : la signification littérale, minimale et indéterminée, à laquelle s'ajoute une signification pragmatique, plus riche et qui corrige en partie le caractère indéterminé de la signification littérale?

J'envisage maintenant une dernière réplique possible du philosophe contextualiste, et qui se traduit par une tentative contraire d'exploiter à fond la thèse d'indétermination. Que dire en effet d'un argument qui, cette fois-ci, s'affiche ouvertement et complètement en faveur de l'indétermination ? Cette version de la thèse de l'indétermination n'admettrait aucun contenu déterminé, que celui-ci provienne du contenu sémantique des phrases elles-mêmes, ou des contenus de pensée associés à leur énonciation dans un contexte donné. Tout en reconnaissant ce fait, le philosophe contextualiste poserait la question : la thèse de l'indétermination ne conduit-elle pas à reconnaître justement que puisque les mots n'ont pas de signification déterminée, ceux-ci voient leur signification fixée par l'usage et donc par le contexte ? Ici, on accepte l'indétermination de la signification des mots et des phrases, et l'on ne prétend pas qu'il faille corriger la situation par le recours à un contexte d'énonciation plus riche. On examine au contraire le versant positif de la théorie wittgensteinienne de l'usage, et on soutient que même si le contexte d'énonciation ne fixe pas pleinement la signification, c'est quand même dans l'usage que les mots acquièrent leur signification. L'indétermination n'est pas appelée à disparaître dans un tel modèle, car on cherche seulement à la neutraliser en partie par un recours à l'usage.

Par « usage ", on ferait ici référence aux occurrences d'un mot. Cellesci viennent enrichir en partie le contenu et permettent de réduire l'indétermination de la signification conventionnelle. Toutefois, si l'argument cherche à exploiter une thèse wittgensteinienne, il devient alors pertinent de faire remarquer qu'il s'agit d'une interprétation pour le moins controversée. Wittgenstein admet l'indétermination des mots et soutient que leur signification est liée à l'usage, mais cela ne veut pas dire qu'il nie l'existence de significations autonomes attachées aux mots et, surtout, que leur signification littérale soit fonction des événements d'énonciation. Ce n'est pas parce que les règles constitutives des jeux sont floues que ceux-ci n'ont pas de règles constitutives. De la même manière, ce n'est pas parce que la signification des mots est indéterminée que ceux-ci n'ont pas de signification conventionnelle 
autonome par rapport au contexte d'énonciation. Dans le cas qui nous occupe, l'argument contextualiste repose en somme sur une confusion entre deux concepts d'usage. Dans la perspective de Wittgenstein, dire que la signification d'un mot est donnée par l'usage veut seulement dire qu'elle dépend des règles qu'une communauté s'est donnée à un moment spécifique au sein d'un dialecte particulier. Il s'agit de règles qui ne sont pas fixées une fois pour toutes, qui n'anticipent pas toutes les applications futures du mot, et qui peuvent varier dans le temps et d'une communauté à l'autre. C'est en ce sens que la signification des mots est liée à l'usage. Néanmoins, ces règles spécifient la signification des expressions types. Cela n'a donc rien à voir avec l'existence d'un lien de nécessité naturelle présumé entre la signification littérale des mots et ce qui se passe dans des événements d'énonciation.

Au terme de cette investigation, je ne sais toujours pas quel est l'argument en faveur du contextualisme. Les arguments discutés plus haut conduisent le philosophe contextualiste à des impasses : à un argument circulaire, à une thèse controversée affirmant le caractère déterminé de la signification, ou à une interprétation erronée du slogan wittgensteinien en vertu duquel la signification d'un mot est donnée dans l'usage. Alors, quel est donc l'argument en faveur du contextualisme? 